Bull. Mater. Sci., Vol. 39, No. 4, August 2016, pp. 1085-1089. (c) Indian Academy of Sciences.

\title{
Spin-wave excitations and magnetism of sputtered Fe/Au multilayers
}

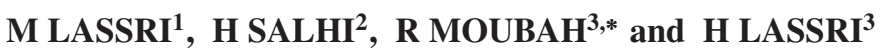 \\ ${ }^{1}$ Centre Régional des Métiers de l'Education et de Formation (CRMEF) de Marrakech Annexe Essaouira, \\ B.P. 44004 Essaouira, Morocco \\ ${ }^{2}$ LMPG, Ecole supérieure de technologie, Université Hassan II de Casablanca, B.P. 5366 Mâarif, Morocco \\ ${ }^{3}$ LPMMAT, Faculté des Sciences Ain Chock, Université Hassan II de Casablanca, B.P. 5366 Mâarif, Morocco
}

MS received 15 September 2015; accepted 15 February 2016

\begin{abstract}
The spin-wave excitations and the magnetism of Fe/Au multilayers with different Fe thicknesses $\left(t_{\mathrm{Fe}}\right)$ grown by RF sputtering were investigated. The temperature dependence of spontaneous magnetization is well described by a $T^{3 / 2}$ law in all multilayers in the temperature range of 5-300 K. Spin-wave theory has been used to explain the temperature dependence of the spontaneous magnetization and the approximate values for the exchange interactions for various $t_{\mathrm{Fe}}$ were obtained. The spin-wave constant $B$ was found to increase linearly with the inverse in the $\mathrm{Fe}$ thickness $\left(1 / t_{\mathrm{Fe}}\right)$. Using the ferromagnetic resonance technique, the change of the anisotropy field $H_{\mathrm{K}}$ as a function of $1 / t_{\mathrm{Fe}}$ was deduced. The spatial distributions of the discrete spin-wave modes were calculated. All the extracted results were in agreement with those determined experimentally and found in the literature.
\end{abstract}

Keywords. Fe/Au multilayers; magnetization; spin-wave excitations; exchange interactions.

\section{Introduction}

Magnetism in ultrathin films has been intensively studied due to the peculiar magnetic properties existing in such systems, with a high potential for spintronic devices [1]. Magnetic multilayers exhibit interesting properties, such as giant magnetoresistance, interlayer exchange coupling and enhancement of magnetic moment of ferromagnetic atoms [2-4]. The period, number of layers and the relative thicknesses of layers affect significantly the multilayer properties, which are in turn sensitive to the microstructure [5-7]. The properties of multilayers are mostly governed by the surface state and hence, the interface plays a key role. The discovery of coupled magnetic behaviour between layer components in different magnetic multilayers has led to an increased interest in two-dimensional systems. In most ferromagnetic materials and below the $T_{\mathrm{C}}$, the temperature dependence of the spontaneous magnetization $M(\mathrm{~T})$ is well described by Bloch's law resulting from the linearized spin-wave theory for bulk ferromagnets [8]. However, for two-dimensional ferromagnets, simulations within the framework of spin-wave theory do not usually predict a $\mathrm{T}^{3 / 2}$ law for the $M(\mathrm{~T})$ curve. In contrast, empirical data have verified the validity of Bloch's law for ultrathin ferromagnetic films in many cases [7]. A possible explanation for this unexpected fact was explained by Mathon and Ahmed [9] who predicted an 'effective $\mathrm{T}^{3 / 2}$ law' to be applicable in a certain temperature range for two-dimensional systems.

\footnotetext{
*Author for correspondence (reda.moubah@ @otmail.fr)
}

On the other hand, the spin-wave excitations are also important parameters in magnetic systems [10-12], which play a crucial role on their magnetic properties at low temperature and they also describe the time scale for the magnetization dynamics. In this paper, the spin-wave excitations and thermal variation of the spontaneous magnetization in $\mathrm{Fe} / \mathrm{Au}$ multilayers as a function of Fe thickness were studied and compared qualitatively and quantitatively with experimental results.

\section{Experimental}

$\mathrm{Fe} / \mathrm{Au}$ multilayers were prepared by $\mathrm{RF}$-sputtering technique. The base pressure was less than $1-2 \times 10^{-7} \mathrm{mbar}$ and the working pressure of Ar was $6.6 \times 10^{-3}$ mbar for deposition. The deposition was carried at room temperature. The thickness was measured in situ using a pre-calibrated quartz monitor. The Fe layer thickness $\left(t_{\mathrm{Fe}}\right)$ was varied from 7 to $72 \AA$ and the Au layer thickness $t_{\mathrm{Au}}$ was fixed at $20 \AA$. The number of bilayers was in the range of 10-20. All the samples were grown on a Au buffer layer of $100 \AA$ thick and covered by a $20 \AA$ thick Au layer to prevent oxidation. In all cases, the first and the last layers were Au. The substrates were floated on glass plates. X-ray diffraction measurements, taken in reflection geometry at both low $\left(2 \theta<10^{\circ}\right)$ and high $\left(30^{\circ} \leq 2 \theta \leq 50^{\circ}\right)$ scattering angles, confirmed the modulated structure and showed a (110) texture for $b c c$ Fe. The $f c c \mathrm{Au}$ buffer layer is seen to have a (111) texture. Magnetization was measured using a vibrating sample magnetometer with an applied magnetic field up to $2 \mathrm{~T}$. 


\section{Results and discussion}

The in-plane $M-H$ hysteresis curves present a rectangular shape (data not shown). The coercivity increases slightly from 3.8 to 9.6 Oe with decreasing $t_{\mathrm{Fe}}$ from 40 to $15 \AA$ at $300 \mathrm{~K}$, which can be seen by the increase of the interface contribution with decreasing film thickness, which should induce an increase in the random anisotropy for thinner $\mathrm{Fe}$ layers. The interlayer exchange coupling strength, $J_{\mathrm{I}}$ can be expressed using the following formula: $J_{\mathrm{I}}=M_{\mathrm{S}} \cdot H_{\mathrm{S}} \cdot t_{\mathrm{Fe}} / 4$, where $M_{\mathrm{S}}$ is the saturation magnetization and $H_{\mathrm{S}}$ is the saturation field [5]. It is found that $J_{\mathrm{I}}$ increases from $5 \times 10^{-3}$ to $10^{-2} \mathrm{erg} \mathrm{cm}^{-2}$, when $t_{\mathrm{Fe}}$ increases from 15 to $40 \AA$.

The magnetization was measured at fields up to $2 \mathrm{~T}$, between 5 and $300 \mathrm{~K}$ using a vibrating sample magnetometer and it is expressed in terms of total volume of the Fe layers. As shown in figure 1 , at $300 \mathrm{~K}$ and $\mathrm{Fe}$ thicknesses ranging from 24 to $72 \AA$, the magnetization is found to be equal to $1700 \pm 80 \mathrm{emu} \mathrm{cm}{ }^{-3}$ but for $t_{\mathrm{Fe}}$ smaller than $24 \AA$, the magnetization increases with the decrease in Fe thickness.

Figure 2 shows the temperature dependence of magnetization for different values of $t_{\mathrm{Fe}}$. It can be noticed that $T_{\mathrm{C}}$ decreases with decreasing $t_{\mathrm{Fe}}$. The decrease of $T_{\mathrm{C}}$ with decreasing $t_{\mathrm{Fe}}$ is evident from the change in the slope of the magnetization curve and can be noted by the reduction in the $\mathrm{Fe}-\mathrm{Fe}$ exchange interaction as a result of finite size effects. The low-temperature magnetization was studied in detail for few samples. For three-dimensional magnetic films, the magnetization has a $T^{3 / 2}$ dependence due to classical spin-wave excitations. In such cases, according to spin-wave theory, the temperature dependence should be written as

$$
\frac{M(5 \mathrm{~K})-M(\mathrm{~T})}{M(5 \mathrm{~K})}=B \mathrm{~T}^{3 / 2},
$$

where $B$ is the spin-wave constant.

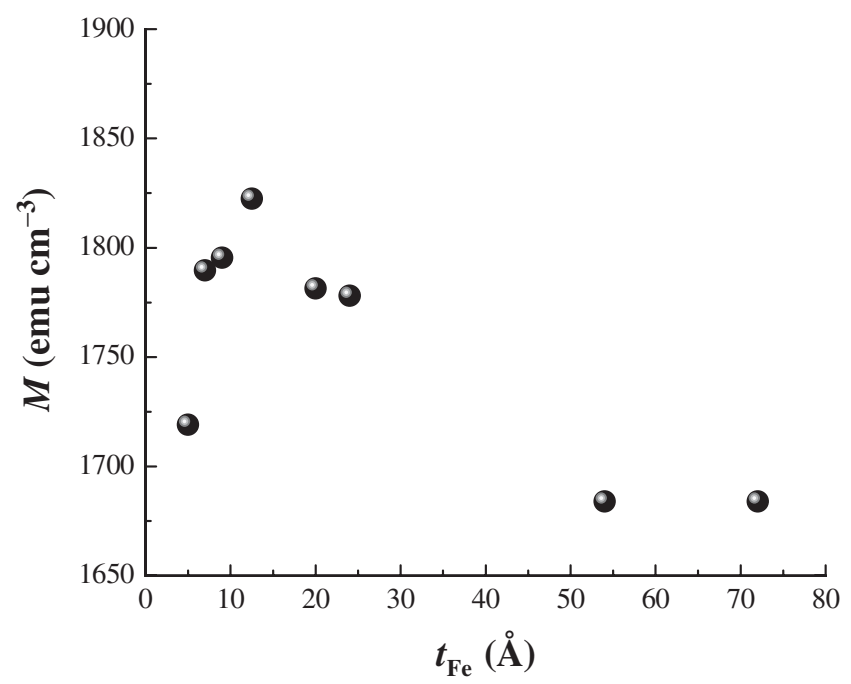

Figure 1. Magnetization vs. Fe thickness recorded at $300 \mathrm{~K}$ for Fe/Au multilayers, the Au thickness was fixed at $20 \AA$.
As observed in figure 2, in all cases, the $M(\mathrm{~T})$ curves are well fitted for temperatures as high as $T_{\mathrm{C}} / 3$ (solid lines). It was noted that $B$ parameter can be expressed as a function of Fe thickness using the following formula $[13,14]$ :

$$
B\left(t_{\mathrm{Fe}}\right)=B_{\text {bulk }}+\frac{B_{\text {surface }}}{t_{\mathrm{Fe}}},
$$

where $B_{\text {Bulk }}$ is the bulk spin-wave constant of Fe and $B_{\text {surface }}$ the $B$ value for the surface contribution. It was found that the interface anisotropy can affect the thickness dependence of the magnetization. The $B\left(t_{\mathrm{Fe}}\right) v s .1 / t_{\mathrm{Fe}}$ curve for samples with Fe thicknesses ranging from 10 to $72 \AA$ is reported in figure 3 . It was found that $B$ decreases from $21 \times 10^{-6}$ to $7.2 \times 10^{-6}$ $\mathrm{K}^{-3 / 2}$ with increasing $t_{\mathrm{Fe}}$ from 10 to $72 \AA$, respectively. The experimental points fit reasonably with the straight line. The extrapolation of the linear fit gives the value of the bulk spinwave constant which is equal to $B_{\text {bulk }}=4.9 \times 10^{-6} \mathrm{~K}^{-3 / 2}$,

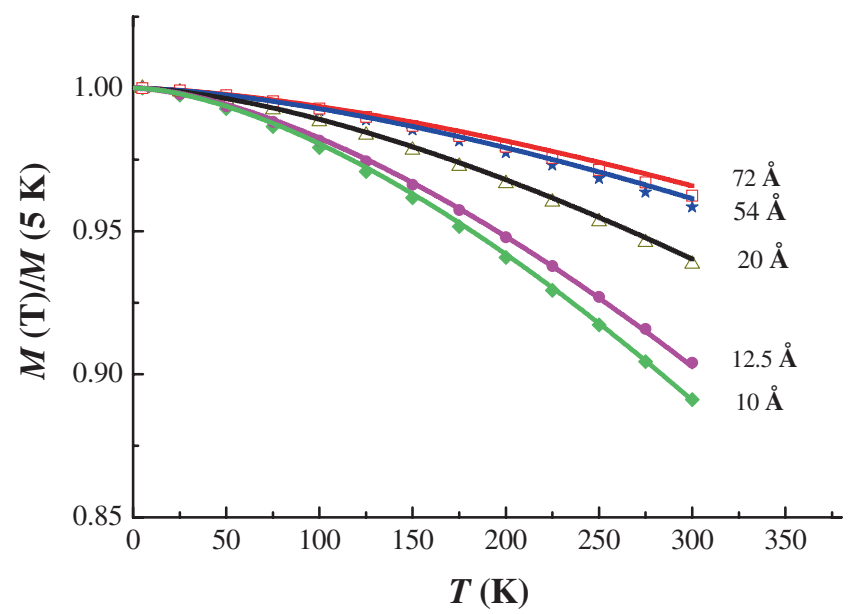

Figure 2. Measured (symbols) and calculated (continuous line) temperature dependence of the normalized magnetization of $\mathrm{Fe} / \mathrm{Au}$ multilayers for different Fe thicknesses.

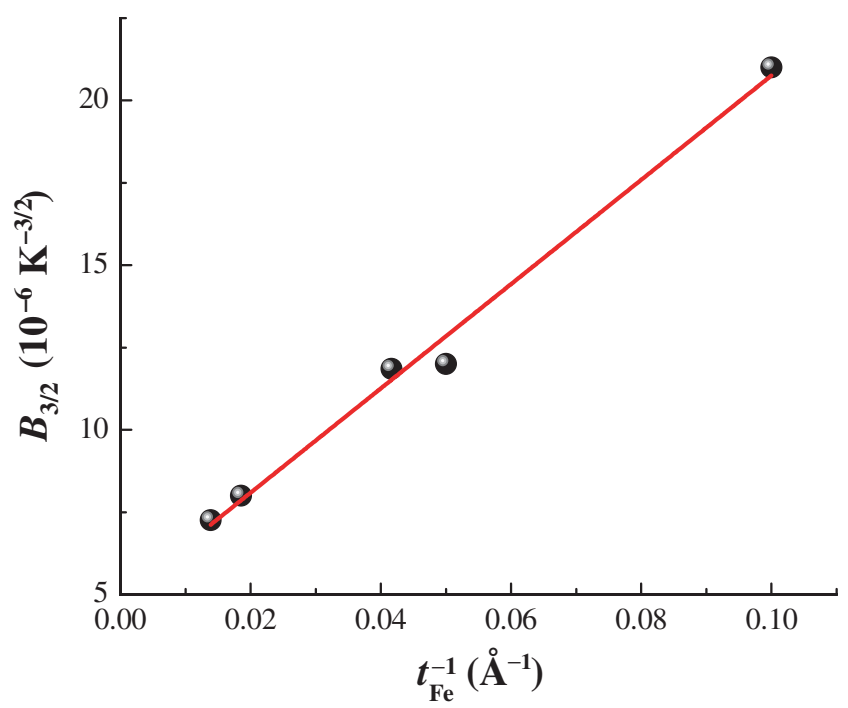

Figure 3. The spin-wave constant $(B)$ as a function of $t_{\mathrm{Fe}}^{-1}$. 
and it is in good agreement with the value obtained for macroscopic-sized samples of bulk bcc Fe [15]. From the slopes of the straight line, the $B$ value on surface, $B_{\text {surface }}$ is deduced to be $1.6 \times 10^{-4} \AA \mathrm{K}^{-3 / 2}$.

To obtain the $g$-factor $(g=2.05 \pm 0.03)$ and effective field magnetization, $4 \pi M_{\text {eff }}$, ferromagnetic resonance measurements with frequencies ranging from 25.09 to $35.73 \mathrm{GHz}$ with the field applied both parallel and perpendicular to the film plane were carried out. The uniaxial anisotropy field, $H_{\mathrm{U}}$, was determined by knowing $M_{\mathrm{S}}$ from the magnetic measurements $\left(H_{\mathrm{U}}=4 \pi M_{\mathrm{S}}\right)$.

According to previous studies [3,13], an interface magnetic anisotropy of multilayers can be seen through the magnetic layer thickness dependence of the perpendicular anisotropy field. If the interface anisotropy mainly increases the first-order anisotropy energy $K_{1}$, then, the perpendicular anisotropy field (excluding the demagnetization term) can be expressed as

$$
H_{\mathrm{K}}=4 \pi M_{\mathrm{S}}-4 \pi M_{\mathrm{eff}}=H_{\mathrm{U}}+2 H_{\mathrm{SA}} / t_{\mathrm{Fe}},
$$

where $H_{\mathrm{U}}$ is the volume anisotropy field and $H_{\mathrm{SA}}$ the surface contribution to the anisotropy field. It was noticed that the perpendicular anisotropy field can also be expressed as $H_{\mathrm{K}}=$ $2 K_{1} / M_{\mathrm{S}} . H_{\mathrm{U}}$ and $H_{\mathrm{SA}}$ can also be written as $H_{\mathrm{U}}=2 K_{\mathrm{U}} / M_{\mathrm{S}}$ and $H_{\mathrm{SA}}=2 K_{\mathrm{S}} / M_{\mathrm{S}}$.

Figure 4 shows the plot of $H_{\mathrm{K}}$ as a function of $1 / t_{\mathrm{Fe}}$. A linear variation of $H_{\mathrm{K}} v s .1 / t_{\mathrm{Fe}}$ can be observed. From the slope of the straight line, the value of the interface anisotropy constant $K_{\mathrm{S}}$ is deduced to be $0.38 \mathrm{erg} \mathrm{cm}^{-2}$ at $300 \mathrm{~K}$. The positive value of $K_{\mathrm{S}}$ indicates that demagnetization energy is still dominating, which means that the perpendicular magnetization is not stabilized in these multilayers.

Theoretical calculations were performed using a model for spin waves in ferromagnetic/nonmagnetic multilayers as described in reference [14]. The basic characteristics may be summarized as follows: the multilayer $\left(\mathrm{Fe}_{n} / \mathrm{Au}_{m}\right)_{q}$ is

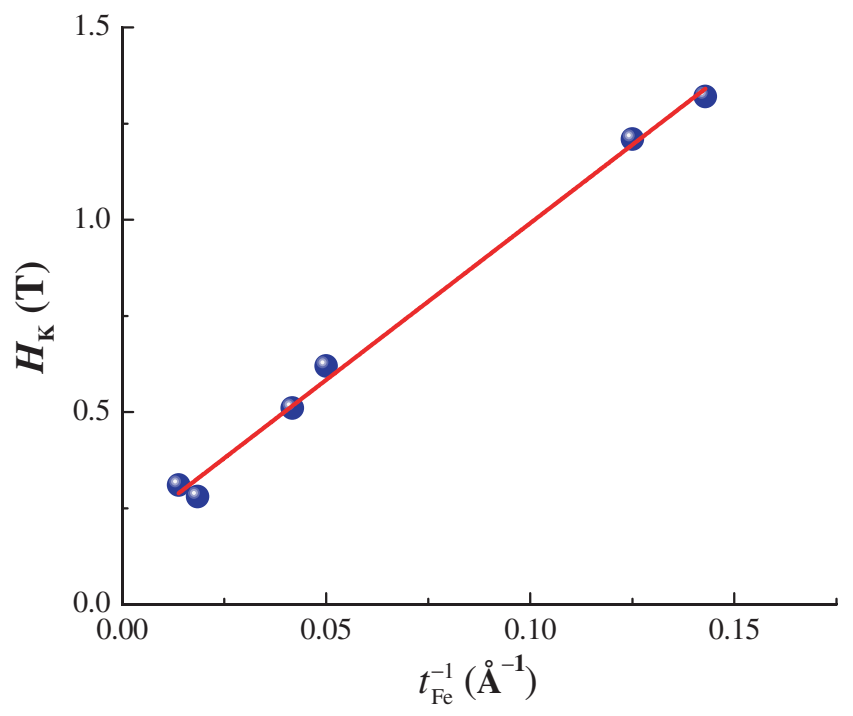

Figure 4. Change of $H_{\mathrm{K}}$ as a function of $t_{\mathrm{Fe}}^{-1}$ for $\mathrm{Fe} / \mathrm{Au}$ multilayers. supported to be formed by an alternate deposition of a magnetic layer $(\mathrm{Fe})$ and nonmagnetic layer $(\mathrm{Au})$. The multilayer is characterized by the number $(q)$ of bi-layers $(\mathrm{Fe} / \mathrm{Au})$. The number of atomic planes in the magnetic and nonmagnetic layers are denoted $n$ and $m$, respectively.

The lattice unit vectors $\left(\vec{e}_{\mathrm{X}}, \vec{e}_{\mathrm{Y}}, \vec{e}_{\mathrm{Z}}\right)$ was chosen, so that $\vec{e}_{\mathrm{Z}}$ is perpendicular to the atomic planes. $\vec{S}_{i \alpha \mu}$ the spin operator of the atom $i(i=1,2, \ldots, N)$ in the plane $\alpha(\alpha=1,2, \ldots$, $n)$ of the magnetic layer $\mu(\mu=1,2, \ldots, q)$ was observed. Furthermore, it was assumed that the multilayer is characterized by a rigid lattice and perfect sharp layer interfaces without structural imperfections (contamination, diffusion, island growth, etc.).

The full Hamiltonian system can be expressed as a summation of two terms

$$
H=H_{\mathrm{e}}+H_{\mathrm{s}} \text {. }
$$

$H_{\mathrm{e}}$ describes the exchange interactions in the same magnetic layer (bulk and surface) as well as the exchange interactions between adjacent magnetic layers which can be written as

$$
\begin{aligned}
H_{\mathrm{e}}= & -J_{\mathrm{b}}\left[\sum_{\langle i \alpha \mu j \alpha \mu\rangle}^{b} S_{i \alpha \mu} S_{j \alpha \mu}+\sum_{\left\langle i \alpha \mu j \alpha^{\prime} \mu\right\rangle} S_{i \alpha \mu} S_{j \alpha^{\prime} \mu}\right] \\
& -J_{\mathrm{s}} \sum_{\langle i \alpha \mu j \alpha \mu\rangle}^{s} S_{i \alpha \mu} S_{j \alpha \mu}-J_{\mathrm{I}} \sum_{\left\langle i \alpha \mu j \alpha^{\prime \prime} \mu^{\prime \prime}\right\rangle}^{I} S_{i \alpha \mu} S_{j \alpha^{\prime \prime} \mu^{\prime \prime}} .
\end{aligned}
$$

$J_{\mathrm{b}}$ and $J_{\mathrm{s}}$ are the bulk and surface exchange interactions. $J_{\mathrm{I}}$ is the interlayer coupling strength which depends on the number $m$ of atomic planes in the non-magnetic layer. $\sum^{b}$ and $\sum^{s}$ are the summations on the sites of bulk and surface layer planes. $\sum^{I}$ is the contribution for the surfaces planes coupled via the nonmagnetic layer. The summation with \langle\rangle denotes the pairs of nearest-neighbour atoms within the same plane or adjacent magnetic planes.

The contribution of the surface anisotropy is estimated by using the following formula:

$$
H_{\mathrm{s}}=D^{\perp} \sum_{i \alpha \mu}^{s}\left(S_{i \alpha \mu}^{\mathrm{Z}}\right)^{2}+D^{\|} \sum_{i \alpha \mu}^{s}\left(\left(S_{i \alpha \mu}^{\mathrm{X}}\right)^{2}-\left(S_{i \alpha \mu}^{\mathrm{Y}}\right)^{2}\right) .
$$

$D^{\perp}$ and $D^{\|}$are the surface anisotropy parameters for the uniaxial out-of-plane and in-plane components, respectively.

In the Holstein-Primakoff formulation [16], the creation and annihilation operators $\left(a_{i \alpha \mu}\right.$ and $\left.a_{i \alpha \mu}^{+}\right)$for each atomic spin are related to the spin operators by

$$
\begin{aligned}
& S_{i \alpha \mu}^{\mathrm{X}}+i S_{i \alpha \mu}^{\mathrm{Y}}=(2 S)^{1 / 2} f_{i \alpha \mu}(2 S) a_{i \alpha \mu}, \\
& S_{i \alpha \mu}^{\mathrm{X}}-i S_{i \alpha \mu}^{\mathrm{Y}}=(2 S)^{1 / 2} a_{i \alpha \mu}^{+} f_{i \alpha \mu}(2 S) .
\end{aligned}
$$

In the framework of non-interacting spin-wave theory, the linear approximation of the Holstein-Primakoff method is sufficient to describe the magnetic behaviour and the correction terms are quite-small at low temperatures $(T<$ $\left.T_{\mathrm{C}} / 3\right)$. As a result, the value of $f_{i \alpha \mu}(2 S)$ can be fixed to 1 [17]. 
The terms $a_{i \alpha \mu}, a_{i \alpha \mu}^{+}$were replaced by the magnon variables $\left(b_{k \alpha \mu}, b_{k \alpha \mu}^{+}\right)$after a two-dimensional Fourier transformation and thus, the following formula was obtained:

$$
\begin{aligned}
H= & H_{0}+A \sum_{\vec{k} \alpha \mu}^{S}\left(b_{\vec{k} \alpha \mu} b_{-\vec{k} \alpha \mu}+b_{\vec{k} \alpha \mu}^{+} b_{-\vec{k} \alpha \mu}^{+}\right) \\
& +\sum_{\vec{k} \alpha \mu}^{S} B_{k} b_{\vec{k} \alpha \mu}^{+} b_{\vec{k} \alpha \mu}+\sum_{\vec{k} \alpha \mu}^{b} C_{k} b_{\vec{k} \alpha \mu}^{+} b_{\vec{k} \alpha \mu} \\
& +\sum_{\vec{k}\left(\alpha \mu, \alpha^{\prime} \mu\right)} D_{k} b_{\vec{k} \alpha \mu}^{+} b_{\vec{k} \alpha^{\prime} \mu} \\
& +\sum_{\vec{k}\left(\alpha \mu, \alpha^{\prime \prime} \mu^{\prime \prime}\right)}^{I} E_{k} b_{\vec{k} \alpha \mu}^{+} b_{\vec{k} \alpha^{\prime \prime} \mu^{\prime \prime}},
\end{aligned}
$$

where

$$
\begin{aligned}
A= & \frac{S}{2}\left(D^{\perp}-D^{\|}\right), \\
B_{k}= & 2 S\left(J_{\mathrm{s}}\left(n^{\|}-\lambda_{k}\right)+J_{\mathrm{b}} n^{\perp}+J_{\mathrm{I}} n^{\uparrow}\right) \\
& +S\left(3 D^{\|}+D^{\perp}\right), \\
C_{k}= & 2 J_{\mathrm{b}} S\left(\left(n^{\|}-\lambda_{k}\right)+2 n^{\perp}\right), \\
D_{k}= & -J_{\mathrm{b}} S \lambda_{\vec{k}}^{\prime}, \\
E_{k}= & -J_{\mathrm{I}} S \lambda_{\vec{k}}^{\prime} .
\end{aligned}
$$

$H_{0}$ is a constant term and $D_{\text {eff }}^{2}=D_{\perp}^{2}+D_{\text {ll }}^{2} . D_{\text {eff }}(K)=K_{\mathrm{S}}$ $a^{2} / k_{\mathrm{B}}$, where $a$ is the lattice constant and $k_{\mathrm{B}}$ the Boltzmann constant.

The coefficients $\lambda_{k}$ and $\lambda_{k}^{\prime}$ depend on the crystallographic structure of the magnetic layer. $n^{\|}$represents the number of nearest-neighbour sites in the same atomic plane, while $n_{\mathrm{S}}^{\perp}$ and $n_{\mathrm{V}}^{\perp}$ are the numbers of surface and volume nearest neighbours in the adjacent plane in the same magnetic layer, respectively. For a given site in the surface plane of the magnetic layer, $n^{\downarrow}$ represents the number of nearest-neighbour sites in the adjacent layer across the nonmagnetic layer. For $b c c$ (110), $n^{\|}=4$ and $n^{\perp}=2$, with the lattice constant $a$ and in the case where the nonmagnetic layer does not disturb the succession order of the magnetic atomic planes $\left(n^{\downarrow}=2\right)$ :

$$
\lambda_{k}=4 \cos \left(a k_{x} \sqrt{ } 2 / 2\right) \cos \left(a k_{y} / 2\right) \text { and } \lambda_{k}^{\prime}=4 \cos \left(a k_{y} / 2\right) \text {. }
$$

The spin system is characterized by $2 n q \times 2 n q$ equations, then the resulting secular equation

$$
\left\{\begin{array}{c}
\left(C_{k}+B_{k}+\omega_{k \alpha \mu}\right) b_{k \alpha \mu}+D_{k} b_{k \alpha^{\prime} \mu} \\
+E_{k} b_{k \alpha^{\prime} \mu^{\prime}}+2 A b_{-k \alpha \mu}^{+}=0, \\
2 A b_{k \alpha \mu}+D_{k} b_{-k \alpha^{\prime} \mu}^{+}+E_{k} b_{-k \alpha^{\prime} \mu^{\prime}}^{+} \\
+\left(C_{k}+B_{k}-\omega_{k \alpha \mu}\right) b_{-k \alpha \mu}^{+}=0 .
\end{array}\right.
$$

We consider the $n \times q$ as positive ones which correspond to the $n \times q$ magnon excitation branches $\omega_{k}^{r}(r=1,2, \ldots$ $n \times q$ ). As can be seen in figure 5 , these branches can be classified into $n$ groups of $q$ quasi-degenerate components

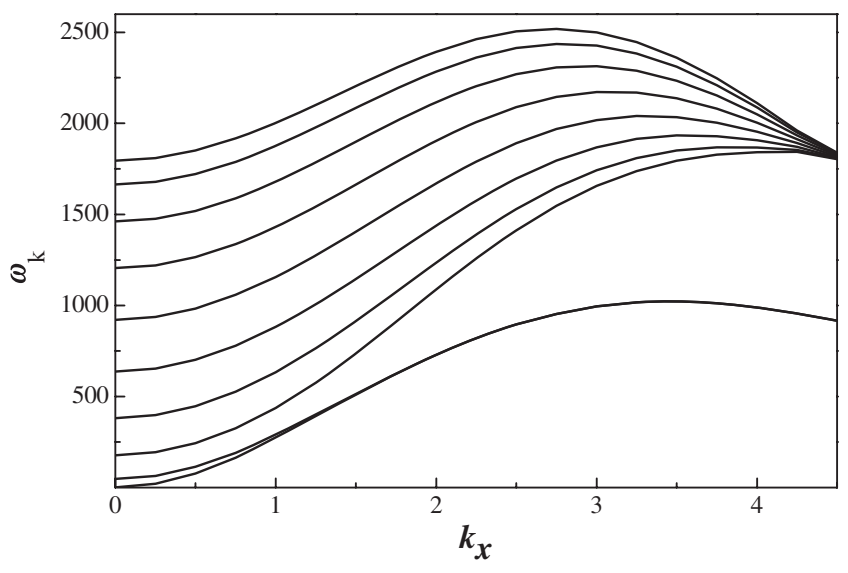

Figure 5. Spin-wave excitation spectrum as a function of $k_{x}$ $\left(k_{y}=k_{x} \sqrt{2}\right)$ for Fe/Au multilayers, in the case of: $q=1, n=10$; $S=1.15 ; J_{\mathrm{b}}=100 \mathrm{~K}, J_{\mathrm{S}}=50 \mathrm{~K} ; D^{\|}=0.9 \mathrm{~K} ; D^{\perp}=-2 \mathrm{~K}$ and $J_{\mathrm{I}}=10^{-2} \mathrm{~K}$.

as expected, in the usual case where $J_{\mathrm{I}}$ remains sufficiently small compared to the effective interlayer exchange strength. The reduced magnetization $v s$. temperature is computed numerically from

$$
m(T)=1-\frac{1}{N_{k} n q S} \sum_{k, r} \frac{1}{\exp \left(\frac{\omega_{k}^{r}}{k_{\mathrm{B}} T}\right)-1} .
$$

The coefficient $N_{k}$ indicates the number of $k$ points taken in the first Brillouin zone. In equation (12), the zero-point fluctuation effects have not been taken into account.

Taken $S=1.15, D^{\|}=0.9 \mathrm{~K}$ and $D^{\perp}=-2 \mathrm{~K}(0.38$ erg $\mathrm{cm}^{-2}$ ), the values of $J_{\mathrm{b}}$ and $J_{\mathrm{S}}$ are found to be equal to $90 \pm 5$ and $45 \pm 5 \mathrm{~K}$, respectively, for all multilayers. The deduced bulk exchange interaction constants are consistent with the expected range for the Fe bulk exchange interaction [18]. The interlayer coupling strength is found to change from $10^{-3}$ to $10^{-2} \mathrm{~K}$, with increasing $t_{\mathrm{Fe}}$ from 10 to $72 \AA$, respectively. We note that different values were reported in the literature for the interlayer coupling strengths as found in refs [19-21]. However, our results remain in the same order but a bit smaller than that reported by Gutierrez et al [22] in $\mathrm{Fe} / \mathrm{Ag}$ multilayer. Compared to the bulk exchange interaction coupling, however, the interlayer coupling is considerably weaker and its effect on the magnetic properties is negligible.

\section{Conclusion}

In conclusion, $\mathrm{Fe} / \mathrm{Au}$ multilayers were prepared by RF sputtering and their spin-wave excitations and magnetic properties were studied. The thermal variation of the magnetization in our multilayers is modelled using spin-wave theory. A simple model has allowed us to obtain numerical estimates of different fundamental constants. The spatial distributions of the discrete spin-wave modes were also determined. Reasonable agreement with the experimental data was reported. 
Finally, this study will be useful to understand the magnetic properties of $\mathrm{Fe} / \mathrm{Au}$ multilayers.

\section{References}

[1] Moubah R, Magnus F, Östman E, Muhammad Y, Arnalds U B, Ahlberg M et al 2013 J. Phys.: Condens. Matter 25416004

[2] Grunberg P, Schreiber R, Pang Y, Brodsky M B and Sower H 1986 Phys. Rev. Lett. 572442

[3] Parkin S S P 1991 Phys. Rev. Lett. 673598

[4] Freeman A J and Wu R 1992 J. Magn. Magn. Mater. 104-107 1

[5] Kesteren H W and van Zeper W B 1991 J. Magn. Magn. Mater. 120271

[6] Krishnan R, Das A and Porte M 1997 J. Magn. Magn. Mater. 16815

[7] Hamouda H, Lassri M, Abid M, Lassri H, Saifaoui D and Krishnan R 2004 J. Mater. Sci.: Mater. Electron. 15395

[8] Bloch F 1930 Z. Phys. 61206

[9] Mathon J and Ahmed S B 1988 Phys. Rev. B 37660
[10] Varshney D, Mansuri I and Khan E 2013 Bull. Mater. Sci. 36 1255

[11] Plakida N M 2015 J. Super. Nov. Magn. 281309

[12] Buitrago I R and Ventura C I 2013 J. Super. Nov. Magn. 26 2303

[13] Rado G T 1982 Phys. Rev. B 26295

[14] Salhi H, Chafai K, Benkirane K, Lassri H, Abid M and Hlil E K 2010 Physica B $\mathbf{4 0 5} 1312$

[15] Korecki I, Przybylski M and Gradmann U 1990 J. Magn. Magn. Mater. 89325

[16] Holstein T and Primakoff H 1940 Phys. Rev. 581098

[17] Dyson F J 1956 Phys. Rev. 1021217

[18] Jiles D 1991 Introduction to magnetism and magnetic materials (Iowa, USA: Ames) p 134

[19] Bland J A C and Heinrich B 1994 (eds) Ultra-thin magnetic structures II (Berlin: Springer)

[20] Bruno P 1994 J. Appl. Phys. 766972

[21] Stoeffler D, Ounadjela K and Gautier F 1991 J. Magn. Magn. Mater. 93389

[22] Gutierrez C J, Qiu Z Q, Wieczorek M D, Tang H, Walker J C and Mercader R C 1991 Hyperfine Interact. 662 